PROCEEDINGS OF THE

AMERICAN MATHEMATICAL SOCIETY

Volume 127, Number 3, March 1999, Pages 801-804

S 0002-9939(99)04565-7

\title{
LENGTHS OF RADII UNDER CONFORMAL MAPS OF THE UNIT DISC
}

\author{
ZOLTAN BALOGH AND MARIO BONK
}

(Communicated by Albert Baernstein II)

\begin{abstract}
If $E_{f}(R)$ is the set of endpoints of radii which have length greater than or equal to $R>0$ under a conformal map $f$ of the unit disc, then cap $E_{f}(R)=O\left(R^{-1 / 2}\right)$ as $R \rightarrow \infty$ for the logarithmic capacity of $E_{f}(R)$. The exponent $-1 / 2$ is sharp.
\end{abstract}

Suppose $\mathbf{D}$ is the unit disc in the plane. A well-known theorem by Beurling [Beu] states that if $f: \mathbf{D} \rightarrow \mathbf{C}$ is conformal, then the set of radii whose images under $f$ have infinite length has vanishing logarithmic capacity. We give a quantitative version of this statement which is asymptotically sharp and improves an estimate by Pommerenke [Pom, p. 215].

Theorem. There exists a universal constant $K>0$ with the following property.

Suppose $f: \mathbf{D} \rightarrow \mathbf{C}$ is a conformal map with $f^{\prime}(0)=1$. If $E_{f}(R)$ is the set of all $\zeta \in \partial \mathbf{D}$ with length $f([0, \zeta)) \geq R>0$, then cap $E_{f}(R) \leq K / \sqrt{R}$.

On the other hand, there exist functions $f$, e.g. the Koebe function, for which cap $E_{f}(R) \geq \frac{1}{2 \sqrt{R}}$ for large $R$.

Our theorem implies that cap $E_{f}(R)=O\left(R^{-1 / 2}\right)$ as $R \rightarrow \infty$ for all conformal maps $f$ of $\mathbf{D}$ and that $1 / 2$ is the best possible constant in this statement.

\section{NotATION AND AUXILIARY RESULTS}

A curve $\gamma: I \rightarrow \mathbf{C}$ is a continuous mapping of an interval $I \subseteq \mathbf{R}$. It is understood that a curve is locally rectifiable. If we speak of a curve in an open set $\Omega$, then we allow the endpoints of the curve to lie on the boundary of the set. A curve $\gamma$ in $\Omega$ connects two sets $A, B \subseteq \bar{\Omega}$, if $\gamma$ has one endpoint in $A$ and one in $B$. We denote by length $(\gamma) \in[0, \infty]$ the euclidean length of $\gamma$.

For the proof of our theorem we need the following version of the GehringHayman theorem (cf. [GH], [Pom, p. 88]).

Theorem A. There is a universal constant $C>0$ with the following property. Suppose $f: \mathbf{D} \rightarrow \mathbf{C}$ is conformal, $\gamma$ is a curve in $\mathbf{D}$ with endpoints 0 and $\zeta \in \partial \mathbf{D}$, and $[0, \zeta)$ is the radius of $\mathbf{D}$ with endpoint $\zeta$. Then

$$
\text { length } f([0, \zeta)) \leq C \text { length }(f \circ \gamma) \text {. }
$$

Received by the editors April 10, 1997 and, in revised form, June 29, 1997.

1991 Mathematics Subject Classification. Primary 30C85.

The first author was supported by the Finnish Mathematical Society.

The second author was supported by TMR fellowship ERBFMBICT 961462.

(C)1999 American Mathematical Society 
The modulus $\bmod \Gamma \in[0, \infty]$ of a family $\Gamma$ of curves in an open set $\Omega$ is defined as

$$
\bmod \Gamma=\inf _{\rho} \int_{\Omega} \rho(z)^{2} d m_{2}(z) .
$$

Here $m_{2}$ is two-dimensional Lebesgue measure and the infimum is taken over all Borel measurable densities $\rho: \Omega \rightarrow[0, \infty]$ that satisfy $\int_{\gamma} \rho(z)|d z| \geq 1$ for all $\gamma \in \Gamma$, where $|d z|$ means integration with respect to euclidean arc-length. The notation for the modulus should indicate which reference set $\Omega$ we consider, but we will suppress this, since it will be clear from the context which $\Omega$ we mean. If $\Gamma_{1}$ is a curve family in some open set $\Omega_{1}, f: \Omega_{1} \rightarrow \Omega_{2}$ is a conformal map and $\Gamma_{2}$ is the curve family in $\Omega_{2}$ consisting of the curves $f \circ \gamma, \gamma \in \Gamma_{1}$, then $\bmod \Gamma_{1}=\bmod \Gamma_{2}$. See [Pom, Ch. 9] for basic properties of the modulus.

We denote the logarithmic capacity of a Borel set $E \subseteq \mathbf{C}$ by cap $E$. For the definition of the logarithmic capacity see [Pom, Ch. 9].

The following statement which relates the concepts of modulus and capacity is needed in the proof of the theorem. It is part of Pfluger's theorem (cf. [Pom, p. $212])$.

Theorem B. Suppose $E$ is a Borel subset of $\partial \mathbf{D}$ and $\Gamma_{E}(\epsilon)$ is the family of all curves $\gamma$ in $\Omega=\{z \in \mathbf{D}: \epsilon<|z|<1\}$ that connect $\{z \in \mathbf{D}:|z|=\epsilon\}$ and $E$. Then for sufficiently small $\epsilon>0$

$$
\operatorname{cap} E \leq \frac{1+\epsilon}{\sqrt{\epsilon}} \exp \left(-\frac{\pi}{\bmod \Gamma_{E}(\epsilon)}\right)
$$

The next lemma states a standard modulus estimate. The constant $2 \pi$ in this inequality is crucial to get the right asymptotic behavior in the theorem. The usefulness of modulus estimates with sharp constants is well-known and dates back to Ahlfors's distortion theorem (cf. [Ahl]).

Lemma. Suppose $\Omega \subseteq \mathbf{C}$ is a region and $\Gamma$ is a family of curves in $\Omega$ which have one endpoint in a compact set $M \subseteq \bar{\Omega}$. Suppose $M$ is contained in a disc of diameter $\delta>0$ centered at the origin. If $L \geq \delta$ and length $\gamma \geq L$ for all $\gamma \in \Gamma$, then

$$
\bmod \Gamma \leq \frac{2 \pi}{\log (1+L / \delta)}
$$

This lemma and its proof are similar to Lem. 3.2 in [BKR].

Proof of the lemma. In addition to our assumptions on $M$ we may assume that there exists at least one rectifiable curve in $\Omega$ which connects a point in $\Omega$ to a point in $M$. For otherwise it is easy to see that $\bmod \Gamma=0$. (Consider test functions $\rho$ which are equal to $\epsilon>0$ on $B \cap \Omega$ where $B$ is some open disc containing $M$ and 0 elsewhere. Let $\epsilon$ tend to 0 .)

For $w \in \Omega$ define $l(w)=\inf _{\gamma} \operatorname{length}(\gamma)$, where the infimum is taken over all curves in $\Omega$ connecting $w$ and $M$. The additional assumption on $M$ implies that $l(w)<\infty$ for all $w \in \Omega$. The function $l$ is continuous on $\Omega$ and satisfies $l(w) \geq|w|-\delta / 2$ for $w \in \Omega$. Moreover, if $\gamma:\left[0, t_{0}\right] \rightarrow \mathbf{C}$ is a curve in $\Omega$ parameterized with respect to arc-length and if $\gamma(0) \in M$, then $l(\gamma(t)) \leq t$ for $t \in\left(0, t_{0}\right]$. 
Define $\rho: \Omega \rightarrow[0, \infty)$ by

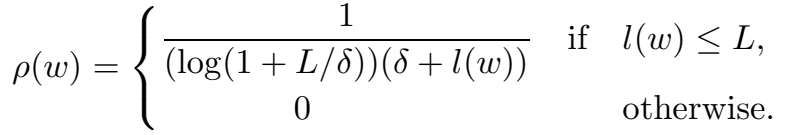

Obviously, the function $\rho$ is Borel measurable and we claim that $\int_{\gamma} \rho(w)|d w| \geq 1$ for all $\gamma \in \Gamma$.

To see this let $\gamma \in \Gamma$ be arbitrary. We may assume that $\gamma: I \rightarrow \mathbf{C}$ has an arc-length parametrization with $I=[0, \operatorname{length}(\gamma)]$ and that $\gamma(0) \in M$. We have $l(\gamma(s)) \leq s$ for all $s \in I \backslash\{0\}$. By assumption length $(\gamma) \geq L$ and so

$$
\int_{\gamma} \rho(w)|d w| \geq \frac{1}{\log (1+L / \delta)} \int_{0}^{L} \frac{d s}{\delta+l(\gamma(s))} \geq \frac{1}{\log (1+L / \delta)} \int_{0}^{L} \frac{d s}{\delta+s}=1 .
$$

Therefore, if $L \geq \delta$

$$
\begin{aligned}
\bmod \Gamma & \leq \int_{\Omega} \rho(w)^{2} d m_{2}(w) \\
& =\frac{1}{[\log (1+L / \delta)]^{2}} \int_{\{w \in \Omega: l(w) \leq L\}} \frac{d m_{2}(w)}{(\delta+l(w))^{2}} \\
& \leq \frac{1}{[\log (1+L / \delta)]^{2}} \int_{\{w \in \mathbf{C}:|w| \leq L+\delta / 2\}} \frac{d m_{2}(w)}{(\delta / 2+|w|)^{2}} \\
& =\frac{2 \pi}{\log (1+L / \delta)}+2 \pi \frac{\log 2-1+\delta /(2 L+2 \delta)}{[\log (1+L / \delta)]^{2}} \\
& \leq \frac{2 \pi}{\log (1+L / \delta)} .
\end{aligned}
$$

The lemma follows.

\section{Proof of the THEOREM}

The idea of the proof is essentially the same as in [Pom, p. 215-216]. A limiting argument is employed in Pfluger's theorem which is related to the concept of reduced extremal distance (cf. [Ahl]). The new ingredients in our proof are the more refined modulus estimate of the lemma and the use of the Gehring-Hayman theorem. The proof will show that for the constant $K$ in the theorem we can take $K=\sqrt{2 C}$ where $C$ is the constant in the Gehring-Hayman theorem.

We use the notation of the theorem and may assume $f(0)=0$. Let $\epsilon \in(0,1)$ be arbitrary. Let $\Gamma_{1}(\epsilon)$ be the family of all curves in $\{z \in \mathbf{D}: \epsilon<|z|<1\}$ connecting $\{z \in \mathbf{D}:|z|=\epsilon\}$ and $E_{f}(R)$. We leave it to the reader to show that the set $E_{f}(R)$ is a countable intersection of open subsets of $\partial \mathbf{D}$. Hence it is a Borel set.

Suppose $\gamma \in \Gamma_{1}(\epsilon)$ and let $z_{0} \in \mathbf{D},\left|z_{0}\right|=\epsilon$, and $\zeta \in E_{f}(R)$ be the endpoints of $\gamma$. Let $\left[0, z_{0}\right]$ be the line segment with endpoints 0 and $z_{0}$. If we join $\left[0, z_{0}\right]$ and $\gamma$, then we get a curve $\tilde{\gamma}$ in $\mathbf{D}$ connecting 0 and $\zeta$. By the Gehring-Hayman theorem and by definition of $E_{f}(R)$

$$
\text { length }(f \circ \tilde{\gamma}) \geq(1 / C) \text { length } f([0, \zeta)) \geq R / C \text {. }
$$

By Koebe's distortion theorem (cf. [Pom, p. 9]), $\left|f^{\prime}(z)\right| \leq(1+5 \epsilon)$ if $|z| \leq \epsilon$ and $\epsilon>0$ is sufficiently small. It follows that for small $\epsilon$

$$
\operatorname{length}(f \circ \gamma) \geq R / C-\left(\epsilon+5 \epsilon^{2}\right)=: L \text {. }
$$


We now apply the lemma for the region $\Omega=f(\mathbf{D} \backslash\{z \in \mathbf{D}:|z| \leq \epsilon\})$, the compact set $M=f(\{z \in \mathbf{D}:|z|=\epsilon\}) \subseteq \bar{\Omega}$ and the curve family $\Gamma_{2}(\epsilon)=\left\{f \circ \gamma: \gamma \in \Gamma_{1}(\epsilon)\right\}$. By Koebe's distortion theorem $M$ is contained in a disc centered at the origin of diameter $\delta=2 \epsilon(1+3 \epsilon)$ for small $\epsilon>0$. It follows that for small $\epsilon>0$

$$
\bmod \Gamma_{1}(\epsilon)=\bmod \Gamma_{2}(\epsilon) \leq \frac{2 \pi}{\log \left(\frac{R / C+\epsilon+\epsilon^{2}}{2 \epsilon(1+3 \epsilon)}\right)} .
$$

Hence Pfluger's theorem implies

$$
\operatorname{cap} E_{f}(R) \leq \liminf _{\epsilon \rightarrow 0} \frac{(1+\epsilon)(2+6 \epsilon)^{1 / 2}}{\left(R / C+\epsilon+\epsilon^{2}\right)^{1 / 2}}=\frac{\sqrt{2 C}}{\sqrt{R}} .
$$

The first part of the theorem follows.

For the second part consider the Koebe function $f(z)=z /(1-z)^{2}, z \in \mathbf{C} \backslash\{1\}$. If $R>1 / 4$ there exists $\phi \in(0, \pi)$ such that $R=1 /\left(4 \sin ^{2}(\phi / 2)\right)$. Since length $f([0, \zeta))$ $\geq|f(\zeta)|$ for $\zeta \in \partial \mathbf{D}$, we have

$$
A=\left\{e^{i \alpha}: \alpha \in[-\phi, \phi]\right\} \subseteq E_{f}(R) .
$$

Since the capacity of the circular $\operatorname{arc} A$ is cap $A=\sin (\phi / 2)$ (cf. [Pom, p. 207]) we obtain cap $E_{f}(R) \geq \frac{1}{2 \sqrt{R}}$. The theorem follows.

\section{ACKNowledgement}

We would like to thank Pekka Koskela who pointed out the sharp constant in the lemma. The results of this paper were obtained while both authors were staying at the Department of Mathematics of the University Jyväskylä. We are glad to acknowledge the hospitality of this institution.

\section{REFERENCES}

[Ahl] L.V. Ahlfors, Conformal invariants: Topics in geometric function theory, McGraw-Hill, New York, 1973. MR 50:10211

[Beu] A. Beurling, Ensembles exceptionnels, Acta Math. 72 (1940), 1-13. MR 1:226a

[BKR] M. Bonk, P. Koskela and St. Rohde, Conformal metrics on the unit ball in euclidean space, to appear in Proc. London Math. Soc.

[GH] F.W. Gehring and W.K. Hayman, An inequality in the theory of conformal mapping, J. Math. Pures Appl. (9) 41 (1962), 353-361. MR 26:6381

[Pom] Ch. Pommerenke, Boundary Behaviour of Conformal Maps, Springer, Berlin, 1992. MR 95b:30008

Universität Bern, Mathematisches Institut, Sidlerstr. 5, CH-3012 Bern, Switzerland

E-mail address: zoltan@math-stat.unibe.ch

Department of Mathematics, University of Jyväskylä, P.O. Box 35, SF-40351 Jyvëskylä, Finland, and Inst. für Analysis, Tech. Univ. Braunschweig, 38106 Braunschweig, Germany

E-mail address: M.Bonk@tu-bs.de 